\title{
Tratamento nutricional dos transtornos
} alimentares

Nutritional Treatment of Eating Disorders

\section{Andréa Romero LatTerza ${ }^{1}$ \\ Karin LOUISE LENZ DUNKeR ${ }^{2}$ \\ Fernanda Baeza Scagliusi ${ }^{3}$ \\ ELISA KEMEN ${ }^{4}$}

\begin{abstract}
Resumo
Pacientes com transtornos alimentares possuem inadequações profundas no consumo, padrão e comportamento alimentar, além de diversas crenças equivocadas sobre alimentação, o que geralmente acarreta piora do estado nutricional. O tratamento nutricional visa a reverter tais alterações e promover hábitos alimentares saudáveis e melhor relação para com o alimento. Os objetivos e características do tratamento diferem para a anorexia nervosa e a bulimia nervosa, contudo, usualmente, a abordagem é dividida em duas fases: a educacional, cujas principais metas são a regularização do hábito alimentar e o aumento do conhecimento nutricional, e a experimental, que visa a propiciar maior reabilitação nutricional e mudanças mais profundas no comportamento alimentar. As evidências existentes sugerem que o tratamento nutricional promove a melhora de tais parâmetros, porém, alguns comportamentos alimentares, como a sensação de incompetência ao lidar com os alimentos, permanecem. Desta forma, mais estudos são necessários para que se possa de fato avaliar a eficácia da abordagem nutricional.
\end{abstract} do Hospital das Clínicas da Faculdade de Medicina da Universidade de São Paulo (HC-FMUSP); mestre em Saúde Pública pela Faculdade de Saúde Pública da USP; professora da Universidade Metodista de São Paulo.

2 Nutricionista, mestre e doutoranda em Nutrição Humana Aplicada pelo Curso Interunidades (FEA/FCF/FSP) da USP: coordenadora de pesquisa do Grupo de Estudos em Nutrição e Transtornos Alimentares (GENTA), bolsista CAPES e Fullbright na Berkeley University (EUA).

3 Nutricionista e coordenadora científica da nutrição/bulimia do Ambulatório de Bulimia e Transtornos Alimentares do Instituto de Psiquiatria, do HC-FMUSP; mestranda em Educação Física pela Escola de Educação Física e Esporte da USP; bolsista CAPES.

4 Nutricionista do Ambulatório de Bulimia e Transtornos Alimentares do Instituto de Psiquiatria do HC-FMUSP.Trabalho desenvolvido no Ambulatório de Bulimia e Transtornos Alimentares do Instituto de Psiquiatria do Hospital das Clínicas da Faculdade de Medicina da Universidade de São Paulo (HC-FMUSP).

Endereço para correspondência: Fernanda Baeza Scagliusi. Laboratório de Nutrição e Metabolismo Aplicados à Atividade Motora, Departamento de Biodinâmica do Movimento do Corpo Humano, Escola de Educação Física e Esporte, Universidade de São Paulo. Av. Prof. Mello Moraes, 65. São Paulo/SP, Brasil. CEP 05508-900. Telefone: (11) 3091-3096. Fax: (11) 38135921, e-mail: fesc@usp.br. 
Palavras-chave: Transtornos alimentares, nutrição, comportamento alimentar, dietoterapia.

\begin{abstract}
Patients with eating disorders have disturbed food intake, food pattern, food behavior, and mistaken beliefs about food and nutrition, which can worsen nutritional status. The nutritional treatment aims to revert such alterations and to promote better food habits and relationship with food. The objectives and characteristics of the treatment are different for anorexia nervosa and bulimia nervosa, but usually the approach has two phases: the educational phase, whose major goals are regularization of food habits and increase of nutrition knowledge; and the experimental phase, whose targets are better nutritional rehabilitation and more complex improvements of nutritional behavior. Evidence suggests that nutritional treatment can ameliorate such parameters, but some inadequate food behaviors remain, such as feeling of incompetence while dealing with food. More studies are needed to evaluate the efficacy of the nutritional treatment.
\end{abstract}

Keywords: Eating disorders, nutrition, food behavior, diet therapy.

\section{Introdução}

Dentro da equipe multidisciplinar que deve tratar do paciente com transtornos alimentares (TA), o nutricionista é capacitado para propor modificações do consumo, padrão e comportamento alimentares, aspectos estes que estão profundamente alterados nos TA.

O tratamento nutricional dos TA é dividido em duas etapas, educacional e experimental. Deve-se conduzir uma detalhada anamnese acerca dos hábitos alimentares do paciente e histórico da doença. É importante avaliar medidas de peso e altura, restrições alimentares, crenças nutricionais e a relação com os alimentos. A educação nutricional abrange conceitos de alimentação saudável, tipos, funções e fontes dos nutrientes, recomendações nutricionais, conseqüências da restrição alimentar e das purgações. Na fase experimental, trabalha-se mais intensamente a relação que o paciente tem para com os alimentos e o seu corpo, ajudando-o a identificar os significados que o corpo e a alimentação possuem (ADA, 1994; Alvarenga, 2001).

O trabalho do nutricionista na área de TA exige habilidades não-inerentes à sua formação, como conhecimentos de psicologia, psiquiatria e das técnicas da terapia cognitivo-comportamental. Deve ser criado um vínculo com o paciente, atuando de forma empática, colaborativa e flexível (Rock e CurranCelentano, 1996).

Um dos princípios do tratamento nutricional dos TA é o uso do diário alimentar. Ele é um instrumento de automonitoração, no qual o paciente registra quais alimentos foram consumidos e a quantidade, os horários e locais das refeições, a ocorrência de compulsões e purgações, a companhia durante as refeições, os sentimentos associados e uma "nota" para o quanto de fome estava sentindo antes de alimentar-se e o quanto de saciedade ele obteve com aquela ingestão. Esse registro faz com que o paciente adquira maior consciência sobre diversos aspectos da sua doença e constantemente exerça disciplina e controle (Story, 1986).

\section{Tratamento nutricional da anorexia nervosa}

As metas do tratamento nutricional na anorexia nervosa (AN) envolvem o restabelecimento do peso, normalização do padrão alimentar, da percepção de fome e saciedade e correção das seqüelas biológicas e psicológicas da desnutrição (APA, 2000). 
O ganho de peso deve ser controlado. É recomendado um ganho de $900 \mathrm{~g}$ a $1,3 \mathrm{~kg} / \mathrm{semana}$ para pacientes de enfermaria e $250 \mathrm{~g}$ a $450 \mathrm{~g} / \mathrm{semana}$ para pacientes de ambulatório (APA, 2000). Todavia, Ornstein et al. (2003) verificaram que um ganho de peso de $720 \mathrm{~g} / 4$ dias era mais seguro, pois não causava a síndrome da realimentação.

Esta síndrome é caracterizada por anormalidades dos fluidos e eletrólitos (principalmente do fósforo) e pode levar a complicações cardiológicas, neurológicas, hematológicas e até à morte súbita. Portanto, a alimentação deve ser cautelosa, com monitoração dos eletrólitos (APA, 2000).

O consumo energético recomendado é de 30 a 40 $\mathrm{kcal} / \mathrm{kg}$ por dia, podendo chegar até 70 a $100 \mathrm{kcal} / \mathrm{kg}$ por dia com a progressão do tratamento (APA, 2000). Segundo Marcason (2002), o valor energético total da dieta não deve ser abaixo de $1.200 \mathrm{kcal} /$ dia. Este aumento gradual pode ajudar a reduzir a ansiedade quanto ao ganho de peso, e permite que o trato gastrointestinal se adapte à realimentação. A proporção de macronutrientes deve ser igual às recomendações para populações saudáveis.

As deficiências de vitaminas e minerais são raras na $\mathrm{AN}$, devido ao uso freqüente de suplementos e à diminuição das necessidades. No entanto, Castro et al, (2004) encontraram deficiências de zinco e ácido fólico em adolescentes com AN que não se reverteram após o tratamento, de tal forma que os autores recomendaram a suplementação destes nutrientes. Su e Birmingham (2004) relatam que as conseqüências da deficiência de zinco são muito semelhantes à $\mathrm{AN}$, e que a suplementação com zinco promove maior ganho de peso e redução da ansiedade e depressão. Em relação ao cálcio, alguns estudos comprovaram os efeitos positivos da suplementação de 1.000 a $2.000 \mathrm{mg} / \mathrm{dia}$ na redução da osteopenia (complicação comum decorrente da AN) (Kent, 2000).

Em alguns pacientes, é extremamente difícil atingir as recomendações nutricionais apenas pela via oral. Nessas ocasiões, a alimentação nasogástrica pode ser recomendada ao invés da intravenosa, que só deve ser utilizada em situações nas quais há risco de vida. A alimentação nasogástrica pode acarretar, contudo, retenção de fluidos, arritmia e falência cardíaca. Apesar de a maioria dos autores não recomendarem a nutrição enteral ou parenteral, Robb et al. (2002) relataram que o uso da sonda nasogástrica noturna pode trazer ganho de peso mais rápido do que somente com a alimentação oral.

O nutricionista participa de todo processo de planejamento das refeições, ajudando o paciente a consumir uma dieta adequada e monitorando o balanço energético, assim como o ganho de peso. Deve-se ajudar o paciente a normalizar o seu padrão alimentar e aprender que a mudança de comportamento deve sempre envolver planejamento e o contato com os alimentos (ADA, 2001).

\section{Tratamento nutricional da bulimia nervosa}

Os objetivos da terapia nutricional na bulimia nervosa (BN) são: diminuir as compulsões, minimizar as restrições alimentares, estabelecer um padrão regular de refeições, incrementar a variedade de alimentos consumidos, corrigir deficiências nutricionais e estabelecer práticas de alimentação saudáveis (ADA, 1994; ADA, 2001; Latner e Wilson, 2001). A abordagem pode ser dividida em alguns pontos chave: a) educação sobre a $\mathrm{BN}$, suas conseqüências e sobre alimentação e nutrição; b) redução da preocupação com peso e aumento da aceitação de seu próprio corpo; c) monitoramento da alimentação por meio do diário alimentar e; d) estabelecimento de um plano alimentar regular (Story, 1986).

É fundamental que o paciente se conscientize que a restrição dietética pode levar a compulsões, e que eles não devem fazer restrições aleatórias, como excluir os alimentos ricos em carboidratos da alimentação (Smith et al., 1999). Não é recomendada a contagem de energia ou pesagem dos alimentos, visto que estes pacientes demonstram extrema atenção na composição dos alimentos e seus valores energéticos (Laessle et al., 1988). É interessante mostrar ao paciente quais são as recomendações de consumo de energia, para que ele possa perceber que o valor energético da maioria das dietas é inferior às necessidades. As conseqüências metabólicas da restrição devem ser explicadas às pacientes.

Pacientes com BN possuem imensa insatisfação corporal, sendo que alguns também têm distorção da imagem corporal (Cash e Deagle, 1997), o que deve ser trabalhado em uma intervenção nutricional. As pacientes que apresentam excesso de peso devem ser conscientizadas que a realização de dietas é incompatível com o tratamento dos transtornos alimentares, ao mesmo tempo. Qualquer alteração de peso deverá ocorrer como resultado da normalização da alimentação e da eliminação das compulsões (ADA, 2001; Rock e Curran-Celentano, 1996).

$\mathrm{O}$ nutricionista deve pesar o paciente regularmente. É comum ocorrer retenção de fluidos quando o paciente reduz o consumo de diuréticos e/ou laxantes e a prática de vômitos. O nutricionista deve explicar que este aumento é transitório e não corresponde a um aumento da adiposidade (Gendall et al., 1997). Também deve ser discutido qual é o peso ideal para o paciente, com base na sua compleição física e histórico de peso. É importante que o paciente compreenda que o peso ideal é determinado em função da sua saúde, e não de padrões de beleza ou exigências pessoais desmedidas.

Poucas pesquisas sobre os efeitos do tratamento nutricional na BN foram conduzidas. Alvarenga (2001) verificou que o tratamento multiprofissional diminuiu a freqüência de compulsões e purgações, sendo que, após o tratamento, $97,5 \%$ das pacientes não preenchiam mais critério diagnóstico. As pacientes passaram a fazer mais refeições sentadas, acompanhadas, 
quietas e a se sentir menos ansiosas e mais tranqüilas durante estas. A preocupação constante com a alimentação e a culpa após comer alimentos "proibidos" diminuíram. Contudo, a maioria permaneceu odiando a sensação de fome, tendo dificuldade com a seleção de alimentos e não acreditando que poderia ter uma alimentação normal e manter um peso normal.

\section{Conclusões}

A participação do nutricional no tratamento dos TAé fundamental, posto que essas doenças implicam alterações profundas no consumo, padrão e comportamento alimentares.

$\mathrm{O}$ tratamento nutricional deve visar à promoção de hábitos alimentares saudáveis, a cessação de comportamentos inadequados (como a restrição, a compulsão e a purgação) e a melhora na relação do paciente para com o alimento e o corpo. Mais estudos acerca do efeito do tratamento nutricional são necessários, assim como a inclusão de variáveis nutricionais nessas pesquisas, como a adequação nutricional, a freqüência de refeições e as diversas manifestações do comportamento alimentar.

\section{Referências bibliográficas}

ADA - American Dietetic Association. - Position of the American Dietetic Association: nutritional intervention in the treatment of anorexia nervosa, bulimia nervosa and binge eating. J Am Diet Assoc 94: 902-7, 1994.

ADA - American Dietetic Association. - Position of the American Dietetic Association: nutrition intervention in the treatment of anorexia nervosa, bulimia nervosa and eating disorders not otherwise specified (EDNOS). J Am Diet Assoc 101: 810- 9, 2001.

Alvarenga, M.S. - Bulimia nervosa: avaliação do padrão e comportamento alimentares. São Paulo, 2001. Tese (Doutorado). Curso Interunidades em Nutrição Humana Aplicada, Universidade de São Paulo.

APA - American Psychiatric Association.- Practice guideline for the treatment of patients with eating disorders. Am J Psychiatry 157: 1-39, 2000.

CASH, T.F.; DeAgLE, E.A. - The nature and extent of body-image disturbances in anorexia nervosa and bulimia nervosa: a meta-analysis. Int $J$ Eat Disord 22: 107-25, 1997.

Castro, J.; Deulofeu, R.; Gila, A.; Puig, J.; Toro, J. - Persistence of nutritional deficiencies after short-term weight recovery in adolescents with anorexia nervosa. Int $J$ Eat Disord 35:169-78, 2004.

Gendall, K.A.; Sullivan, P.E.; Joyce, P.R.; Carter, F.A.; Bulik, C.M. - The nutrient intake of women with bulimia nervosa. Int J Eat Disord 21: 115-27, 1997.

KENT, H. - BC's eating disorders program looks toward outpatient model. CMAJ 162: 684, 2000.
Laessle, R.G.; Schwelger, U.; Daute-Herold, U.; SchWeiger, M.; Fichter, M.M.; PIRKE, K.M. - Nutritional knowledge in patients with eating disorders. Int J Eat Disord 7: 63-73, 1988.

LATNER, J.D.; Wilson, T. - Cognitive-behavioral therapy and nutritional counseling in the treatment of bulimia nervosa and binge eating. Eating Behaviors 1: 3-21, 2000.

MARCASON, W. - Nutrition therapy and eating disorders: what is the correct calorie level for clients with anorexia? $J$ Am Diet Assoc 102: 644, 2002.

Ornstein, R.M.; Golden, N.H.; JaCobson, M.S.; Shenker, I.R. Hypophosphatemia during nutritional rehabilitation in anorexia nervosa: implications for refeeding and monitoring. J Adolesc Health 32: 83-8, 2003.

RobB, A.S.; Silber, T.J.; Orrel-Valente, J.K.; Valadez-Meltzer, A.; ElLIS, N.; Dadson, M.J.; ChATOOR, I. - Supplemental noctural nasogastric refeeding for better short-term outcome in hospitalized adolescent girls with anorexia nervosa. Am J Psychiatry 159: 1347-353, 2002.

Rock, C.L.; Curran-Celentano, J.- Nutritional management of eating disorders. Psych Clin North Am 19: 701-13, 1996.

Smith, C.F.; Williamson, D.A.; Bray, G.A.; Ryan, D.H. - Flexible vs. rigid dieting strategies: relationship with adverse behavioral outcomes. Appetite 32. 295-305, 1999.

StoRy, M. - Nutrition management and dietary treatment of bulimia. J Am Diet Assoc 86: 517-9, 1986.

Su, J.C.; Birmingham, C.L. - Zinc supplementation in the treatment of anorexia nervosa. Eat Weight Disord 7: 20-2, 2002 